\title{
Chemistry significant learning to reduce the failure of students in High School using LKT
}

\author{
DOI: $10.46932 /$ sfjdv3n1-098
}

Received in: Jan 30st, 2021

Accepted in: Feb 1th, 2022

Dr. Néstor Arroyo Ayala

Doctor en Ciencias de la Educación por la Universidad Contemporánea de las Américas.

Catedrático de la Dirección General de Educación Tecnológica Agropecuaria y Ciencias del Mar -

CBTA No 181, en el área de Ciencias Experiméntales.

Maravatío, Michoacán, México

E-mail: nestorarroyo181@ dgetaycm.sems.gob.mx

\section{Dr. David Jesús Becerra Solís}

Doctor en Ciencias de la Educación por la Universidad Contemporánea de las Américas.

Catedrático de la Dirección General de Educación Tecnológica Agropecuaria y Ciencias del Mar CBTA No 181, en el área de Ciencias Experiméntales.

Maravatío, Michoacán, México

E-mail: becerra_dj@hotmail.com

\section{Dr. Antonio Cruz Núñez}

Doctor en Ciencias de la Educación por la Universidad Contemporánea de las Américas.

Catedrático de la Dirección General de Educación Tecnológica Agropecuaria y Ciencias del Mar CBTA No 181, en el área de Informática.

Maravatío, Michoacán, México

E-mail: acn60@hotmail.com

\section{Mtro. José Manuel Gómez Soto}

Maestro en Pedagogía por el Instituto Michoacano de Ciencias de la Educación.

Catedrático de la Dirección General de Educación Tecnológica Agropecuaria y Ciencias del Mar -

CBTA 181, en el área de Comunicación.

Maravatío, Michoacán, México.

E-mail: josegomez181@dgetaycm.sems.gob.mx

\footnotetext{
ABSTRACT

Failure in the area of science is one of the main obstacles that students face, and can even lead them to drop out, therefore, the present work seeks to reduce the failure rates of young people of the High School in the subject of chemistry.

Iintervention strategy was implemented, which consisted of 3 didactic sequences for the teaching of Chemistry, using Learning and Knowledge Technologies (LKT) as the main tool, this, in students of a High School Center in the State of Michoacán, México.

The results showed that the LKT in the teaching of Chemistry, allow a greater number of students to achieve significant learning in the concepts of Atom, Atomic Structure and Chemical Bond, compared to teaching methods that did not include the LKT, due to the above, These technologies could be considered to decrease failure rates in the High School.
} 
Keywords: School failure, Significant learning, LKT.

\section{INTRODUCCIÓN}

Uno de los retos que enfrenta la Educación Media Superior, es el alto índice de deserción, el cual ronda el 30\%. Anualmente entre 600,000 y 700,000 alumnos, abandonan sus estudios.

De acuerdo a la Secretaría de Educación Pública SEP, (2018), los factores de abandono escolar son cuatro; causas económicas 38\%, motivos escolares-institucionales $41 \%$, motivos familiares $5 \%$ y el $16 \%$ restante, otras situaciones diversas.

La importancia de que todos los jóvenes concluyan su EMS, es que esta tiene un impacto positivo a nivel social, pues representa el vínculo entre el sector laboral técnico y el sector universitario. De acuerdo a Tuirán, (2012), los estudiantes de Nivel Medio Superior, tienen altas expectativas de mejora social y económica a través de la vía profesional. Aunado a esto, en la última década, se ha observado un incremento de hechos violentos, donde los involucrados cuentan con escolaridad baja.

Debido al impacto que tiene la reprobación en el NMS, es necesario identificar las causas académicas de la reprobación con la finalidad de disminuirla y no sufrir así, los efectos negativos que esta tiene, por ello, en el presente estudio, se identificaron las causas por las cuales lo jóvenes reprueban la EMS; falta de comprensión a los docentes, aburrimiento durante el desarrollo de las clases, poca o nula utilización de material didáctico digital. Con esta información, se diseñó y se implementó una estrategia de intervención para disminuir los índices de reprobación en la asignatura de Química, utilizando como herramienta principal, las Tecnologías del Aprendizaje y Conocimiento, con estudiantes de primer año de un Centro de Bachillerato Tecnológico del Estado de Michoacán.

\section{DESCRIPCIÓN DEL MÉTODO}

\subsection{DIAGNÓSTICO DEL PROBLEMA.}

Se realizó análisis histórico documental de indicadores académicos del plantel de 5 años previos al 2019, para observar la tendencia de reprobación en las diferentes áreas del conocimiento, identificando que, durante este periodo de tiempo, se han mantenido de forma general, índices de reprobación cercanos al 30\%, lo cual representa un serio problema, ya que la reprobación es uno de los factores descritos como causantes de deserción escolar. 


\subsection{DELIMITACIÓN DEL PROBLEMA Y UNIVERSO DE TRABAJO.}

Se analizaron los resultados del pos test de ingreso al NMS, publicados en el portal de la SEMSCOSDAC. Los resultados mostraron índices de reprobación altos en las áreas de ciencias experimentales, en comparación con las áreas de habilidad lectora y habilidad matemática. Dentro del área de ciencias se consideran las asignaturas de Física, Biología y Química, en esta última evidenciaron severas deficiencias en la comprensión de los contenidos de la signatura, esta tendencia coincidió, con el análisis histórico de indicadores académicos del plantel, donde mostró también el mayor índice de reprobación.

Según el diseño curricular del Bachillerato Tecnológico, la Química se imparte en el primer y segundo semestre, por ello, el universo de trabajo, fueron 365 estudiantes cursantes del primer año.

\subsection{DISEÑO DE LA ESTRATEGIA DE INTERVENCIÓN.}

Se realizaron una serie de encuestas, la primera dirigida a identificar las causas de la reprobación, el instrumento utilizado, contenía tópicos relacionados a causas familiares, económicas, personales y académico-institucionales, esta se aplicó a 216 alumnos que tuvieron al menos una asignatura reprobada en el curso de los tres años de bachillerato del plantel.

Como se muestra en la Figura 1, los estudiantes refieren que les gusta la escuela a), que mayoritariamente no sufre acoso escolar b), que su familia los apoya para estudiar c) y que en términos generales su situación económica es de buena a regular d), por lo cual estos factores se descartan como causantes de reprobación. Por el contario, el 50\% de alumnos encuestados refieren que se quedan con dudas al momento de la explicación docente e), y creen que las causas por las cuales están reprobando son f), la falta de entrega de trabajos, horas de estudio insuficientes, falta de interés, es decir, factores directamente relacionados al aspecto académico.

Figura 1. Resultados de encuesta para identificar las causas de reprobación.

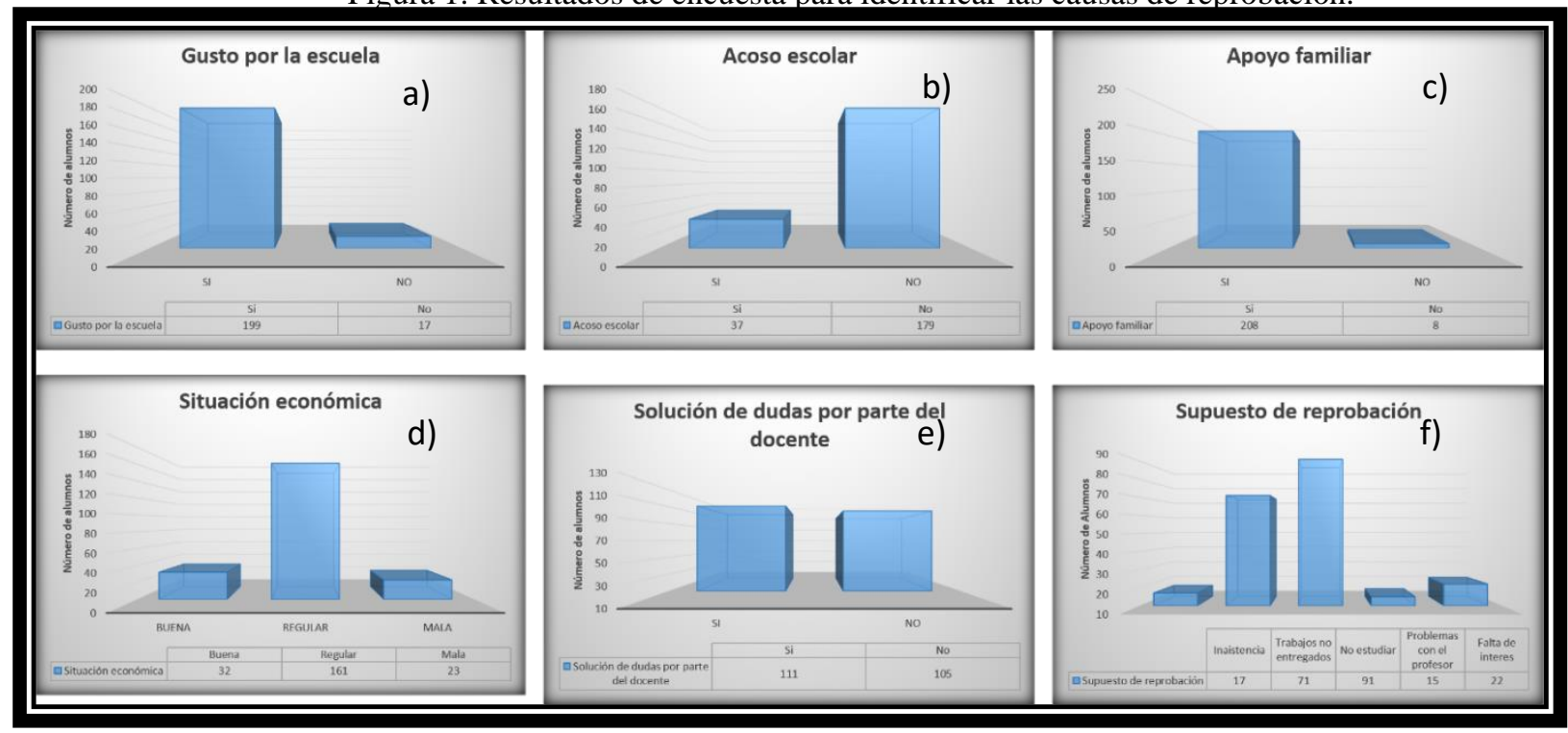


En una segunda etapa, se detectaron núcleos problemáticos de química que dificultan el aprendizaje, esto, a través de análisis y discusiones en equipos heterogéneos con 365 estudiantes de primer grado, donde cada equipo seleccionaba tres contenidos que consideraran realmente complejos de aprender y los puntuaran de menor a mayor dificultad. El Cuadro 1, muestra los contenidos que los estudiantes consideraron más difíciles de comprender; configuración electrónica, la estructuración de la tabla periódica, estructura atómica y enlace químico, la complejidad de dichos contenidos radica en el nivel de abstracción necesario para entender los procesos químicos a nivel de partículas.

\begin{tabular}{l|l} 
Cuadro 1. Núcleos problemáticos para el aprendizaje de la Química. \\
Contenido de Química & 20 \\
\hline Enlace químico & 55 \\
Tabla periódica & -- \\
Propiedades de la materia & 95 \\
Configuración electrónica & 10 \\
Nomenclatura de compuestos inorgánicos & -- \\
Clasificación de la materia & 35 \\
Estructura atómica & 10 \\
Química orgánica & 15 \\
Reacción química &
\end{tabular}

Por último, se encuestó a cuatro docentes que históricamente han sido titulares de las asignaturas de Química, con la finalidad de identificar áreas de oportunidad en el proceso de enseñanza aprendizaje, el cuestionario aplicado, estaba dirigido a determinar si los docentes desarrollaban su clase bajo el modelo constructivista, dividido en los momentos de apertura, desarrollo y cierre, el grado de motivación e interés que mostraban los estudiantes en el desarrollo de las sesiones, así como la frecuencia de utilización de material didáctico y las características de este, encontrando que, 1. el desarrollo de las sesiones se da respetando los momentos de apertura, desarrollo y cierre según el modelo constructivista,2. los docentes refieren que no existe interés de parte de la mayoría de los alumnos e incluso detectan apatía, 3, los docentes no utilizan material didáctico que se refiera, a simuladores, apoyos visuales, material interactivo, ni al menos los video proyectores presentes en las aulas.

\subsection{IMPLEMENTACIÓN DE LA ESTRATEGIA DE INTERVENCIÓN.}

La estrategia de intervención consistió en dos secuencias didácticas, desarrolladas con todos los alumnos del primer semestre, cada secuencia didáctica contempló cuatro horas de trabajo durante cuatro semanas, están secuencias contemplaron los contenidos, de propiedades de la materia, modelo atómico, estructura atómica, configuración electrónica y tabla periódica, abordados, desde la utilización de las TAC; simuladores de la Universidad de Colorado, páginas interactivas IPN y UNAM, así como proyectores táctiles interactivos. 


\section{COMENTARIOS FINALES}

\subsection{RESULTADOS Y DISCUSIÓN}

\subsubsection{Estrategia de intervención}

El aprendizaje significativo, implica construir y anclar nuevos saberes sobre los ya existentes, siempre y cuando estos se hayan logrado a partir de la interacción consciente del alumno, de su participación con todos los sentidos, de su motivación. Ausubel, Novak y Hanesian explican que "la esencia del aprendizaje significativo reside en el hecho de que las ideas están relacionadas simbólicamente y de manera no arbitraria (no al pie de la letra) con lo que el alumnado ya sabe" Ballester, (2002). Los resultados de las encuestas realizados a estudiantes y docentes, mostraron que los factores detonantes de la reprobación, se relacionaban a situaciones académicas, por ello, la estrategia de intervención consistió en una secuencia didáctica enfocada a desarrollar aquellos contenidos de la asignatura considerados por los alumnos como "núcleos problemáticos de Química que dificultan el aprendizaje", los cuales fueron: 1. Configuración electrónica, 2. Estructura atómica y 3. Tabla periódica.

Con la finalidad de evitar el aburrimiento y la falta de interés referida tanto por estudiantes como por docentes de la asignatura, se hizo una puntual selección de recursos digitales que contribuyeran a la construcción del proceso enseñanza aprendizaje, es decir, utilizando las TAC; páginas interactivas, simuladores y blogs, esto se muestra en el Cuadro 2.

Cuadro 2. Diseño general de estrategia de intervención basado en el uso de las TAC.

\begin{tabular}{|c|c|c|}
\hline $\begin{array}{l}\text { Aprendizaje } \\
\text { de Química }\end{array}$ & Actividad & \\
\hline 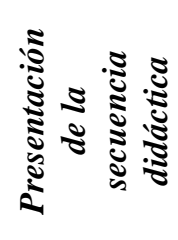 & $\begin{array}{l}\text { Se dio a conocer a los alumnos el Blog de la } \\
\text { Química, en el, los alumnos encontraran } \\
\text { información sobre los contenidos a desarrollar, } \\
\text { instrumentos de evaluación,, así como ligas de } \\
\text { acceso a los diferentes recursos digitales a utilizar } \\
\text { en el desarrollo de la secuencia didáctica. }\end{array}$ & $\begin{array}{l}\text { Blog de la química } \\
\text { http://www.quimi } \\
\text { cacbta181.blogsp } \\
\text { ot.mx } \\
\text { Página interactiva, }\end{array}$ \\
\hline 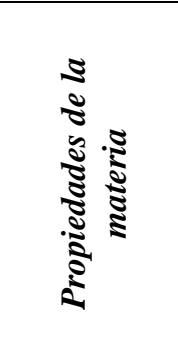 & $\begin{array}{l}\text { A través de a página interactiva, Materia-IPN, } \\
\text { se fueron desarrollando los contenidos } \\
\text { específicos de Propiedades de la materia, } \\
\text { Estados de agregación, mezclas y compuestos, } \\
\mathrm{y} \text { Ley de conservación de la materia. En } \\
\text { seguida, }\end{array}$ & $\begin{array}{l}\text { Materia IPN } \\
\text { https://www.aev.c } \\
\text { gfie.ipn.mx/Mater } \\
\text { ia_quimica/temas/ } \\
\text { tema1/intro1.html } \\
\text { Página interactiva, } \\
\text { Construye un } \\
\text { átomo UNAM }\end{array}$ \\
\hline
\end{tabular}




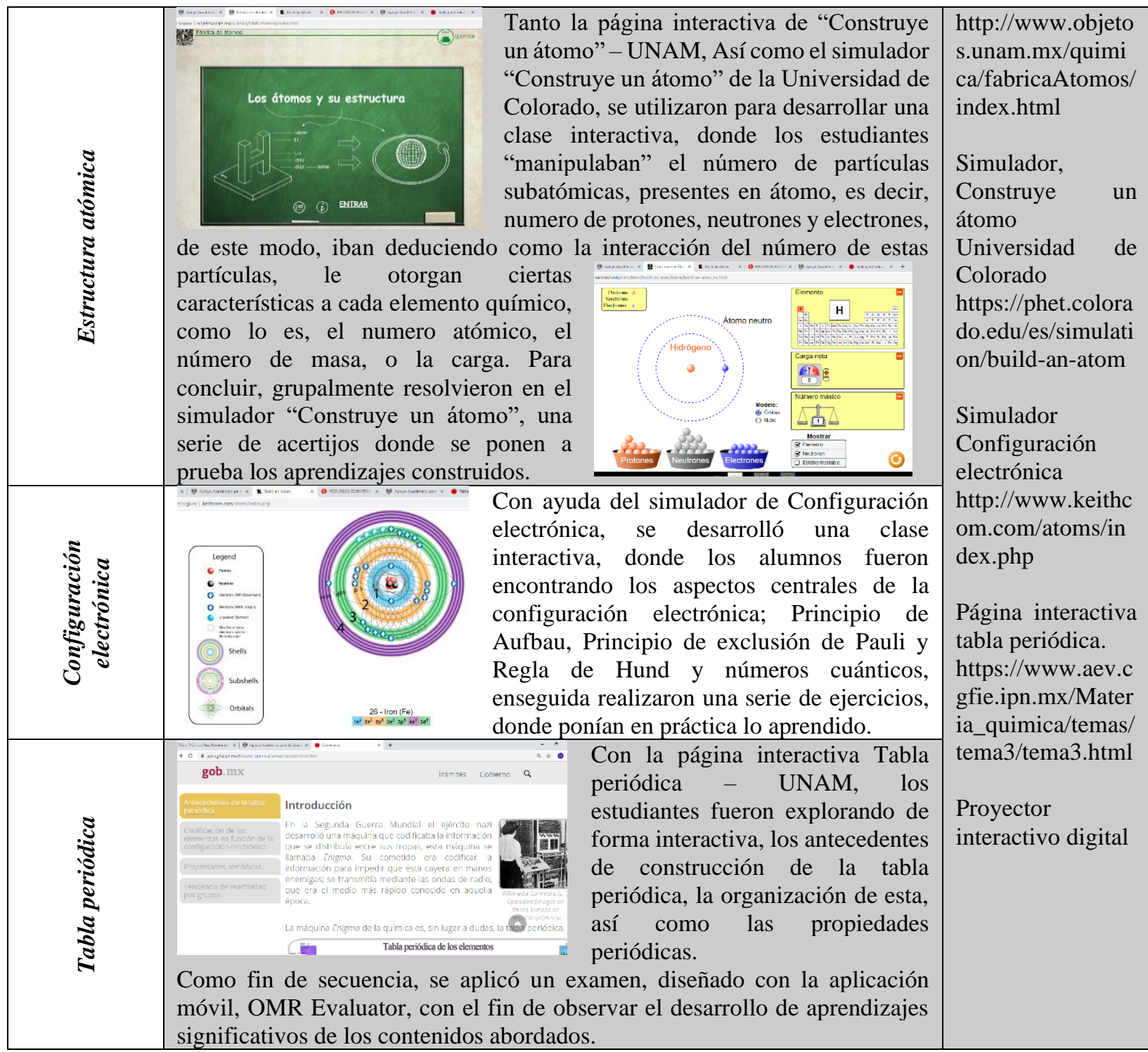

\subsubsection{Implementación de la estrategia de intervención}

El primer resultado apreciable de la implementación de esta estrategia de intervención fue, el cambio de actitud, interés, disposición y participación por parte de los alumnos, como se muestra en la Figura 2, los alumnos permanecen receptivos y participativos, se percibe alegría, e incluso asombro, estas reacciones son un claro ejemplo de lo que Ausubel llama aprendizaje significativo, pues a diferencia de la forma clásica de aprender que es por repetición y memorización, aquí se observa, la interacción alumno - alumno y alumno - docente, con lo cual existe una retroalimentación, además, parten de algo objetivo, de algo que ellos están observando y manipulando. 
Figura 2. Implementación de estrategia de intervención basada en el uso de las TAC.

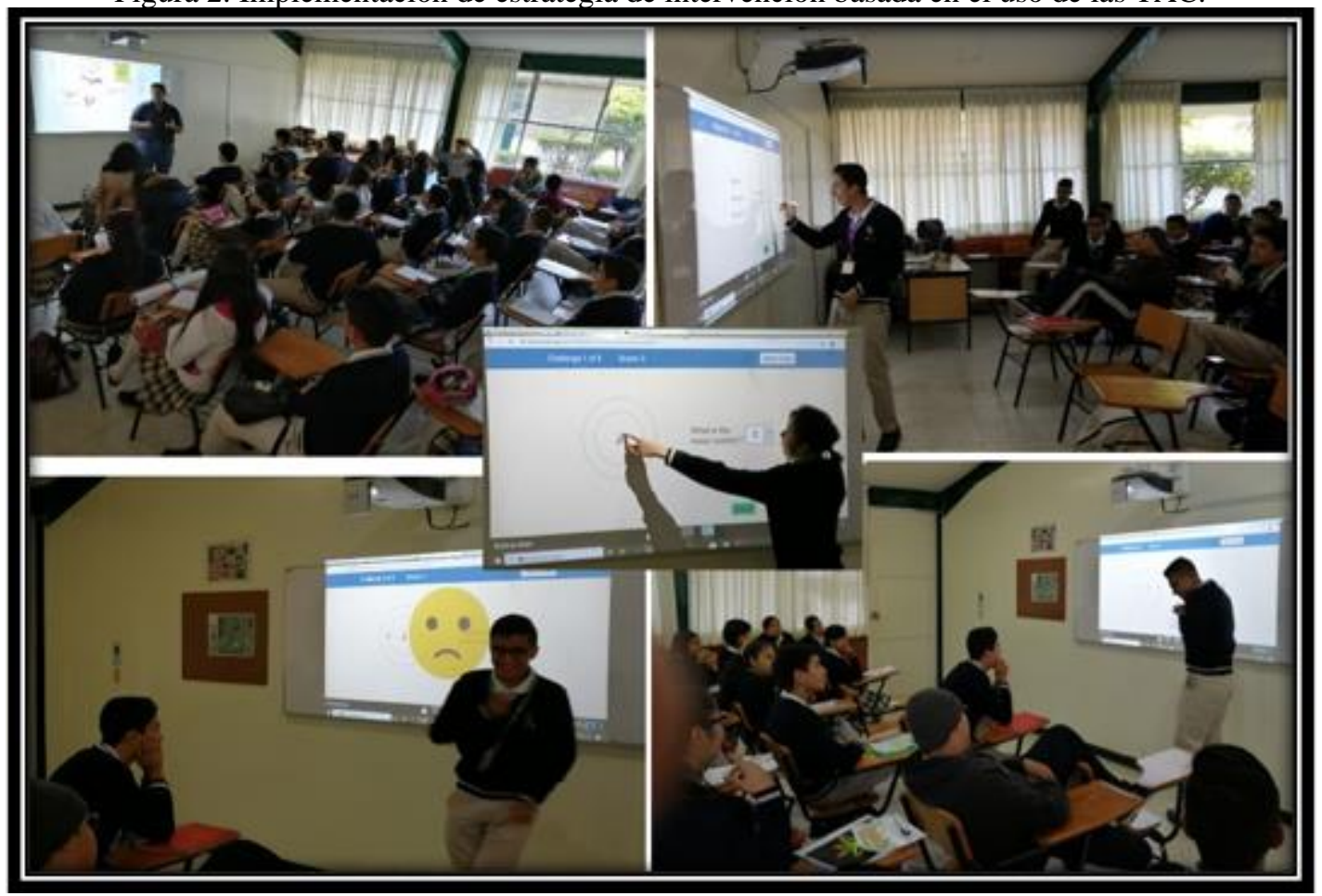

En este contexto, se puede decir que la complejidad de la Química radicaba, en la incapacidad de darle un sentido a los contenidos de esta asignatura. Pedirle a un alumno, que imagine la estructura de un átomo, que comprenda la existencia de niveles y subniveles de energía y que en ellos se encuentran partículas llamadas electrones, solo porque su profesor se los ha dicho, suele ser poco funcional, en cambio con el uso de las paginas multimedia, así como los diferentes simuladores, los alumnos tuvieron un aprendizaje vivencial, práctico, un aprendizaje que terminará siendo significativo.

\subsubsection{Reprobación tras la implementación de la estrategia de intervención}

Utilizando la aplicación móvil OMR Evaluator, se diseñó un examen para evaluar el logro de aprendizajes de la estrategia de intervención, este consistió en diez reactivos de opción múltiple, enfocados al proceso reflexivo, al análisis, más que a la parte memorística del conocimiento.

Después de la aplicación de este examen en los nueve grupos de primer semestre, se observó una aprobación promedio del $60 \%$, la cual resulta ser muy alta, en comparación con los resultados obtenidos de la evaluación de los mismos contenidos durante el año 2018, los cual rondaban el 30\% de aprobación. Estos resultados tan diferentes en años consecutivos, no significan que los procesos de enseñanza aprendizaje fueran tradicionalistas, pues en el plantel, se ha venido trabajando bajo el modelo constructivista, con el impulso del trabajo colaborativo y la socialización, con el fin de sentar en las relaciones sociales el andamiaje para desarrollar en los estudiantes el razonamiento, la comprensión y el pensamiento crítico (Piaget,1985; Bruner,2000 citador por Pimienta, 2007). 
La explicación apropiada a estos resultados, es la utilización de las TAC en los procesos de enseñanza, debemos tener en cuenta que estamos educando a jóvenes del siglo 21, con una habilidad enorme para el uso de los medios digitales, intentar instruirlos, con modelos del siglo pasado, pareciera no ser la mejor opción.

\section{CONCLUSIONES}

La utilización de las Tecnologías del Aprendizaje y el Conocimiento, permiten motivar el interés por aprender y contribuyen a la construcción de aprendizajes significativos en jóvenes estudiantes. Utilizar estas herramientas durante el desarrollo de secuencias didácticas, permitió además disminuir los índices de reprobación en la asignatura de Química, lo cual sugiere que pueden ser una buena alternativa, para evitar que los alumnos reprueben y eventualmente abandonen sus estudios en el Nivel Medio Superior. 


\section{REFERENCIAS}

Ballester, A. (2002). El aprendizaje significativo en la práctica. España: Boletín de Estudios de Investigación.

Pimienta, J. (2007). Metodología constructivista. México: Pearson Educación.

SEP. (2018). Reporte de la encuesta nacional de deserción escolar en la Educación Media Superior. COPEEMS.

Tuirán, A. (2012). La educación superior en México 2006 - 2012 Un balance inicial. Campus Milenio.

Arguedas, I. y Jiménez, F. (2007). Factores que promueven la permanencia de estudiantes en la educación secundaria. Revista Electrónica Actualidades Investigativas en Educación, 7 (3). 1-36.

Ezpeleta, J. (2004). Innovaciones educativas. Reflexiones sobre los contextos en su implementación. Revista Mexicana de Investigación Educativa, vol. IX, no. 21, pp. 403-424

González, A. (2002), reflexiones sobre los factores que influyen a la deserción escolar del adolescente de México, D.F. Universidad Pedagógica Nacional.

Gutiez. P. (2000) influencia de la familia en la integración escolar, revista de orientación pedagógica Bordón vol. 41, No. 3.

López V.L., Beltrán S.A. (2012) Causas de Deserción Escolar en Estudiantes de Escuelas Medio Superior, Revista Electrónica “ Pistas Educativas”, núm. 100, pags. 112-125. 